\title{
Partidos Políticos y Satisfacción con la Democracia "Una mirada desde la institucionalidad de América Latina"
}

\author{
Cabrera Benavides, Lucero Liliana \\ Partidos Políticos y Satisfacción con la Democracia "Una mirada desde la institucionalidad de América Latina" \\ Cuadernos de $\mathrm{H}$ ideas, vol. 13, núm. 13, 2019 \\ Universidad Nacional de La Plata, Argentina \\ DOI: https://doi.org/10.24215/23139048e030
}

Esta obra está bajo una Licencia Creative Commons Atribución-NoComercial-Compartirlgual 4.0 Internacional. 
Artículos

\title{
Partidos Políticos y Satisfacción con la Democracia “Una mirada desde la institucionalidad de América Latina”
}

\author{
Political Parties and Satisfaction with Democracy «A look from the institutional framework of Latin America»
}

Lucero Liliana Cabrera Benavides

DOI: https://doi.org/10.24215/23139048e030

Facultad Latinoamericana de Ciencias Sociales, Ecuador

luceroliliana08@hotmail.com

(D) http://orcid.org/0000-0002-7597-9693

Recepción: 21 Mayo 2019

Aprobación: 20 Diciembre 2019

\section{RESUMEN:}

El artículo se desarrolla a partir de la pregunta de investigación: ¿Cuál es la incidencia de la satisfacción con la democracia en la confianza hacia los partidos políticos en América Latina, 2017? Se aborda a partir de datos de la encuesta anual de opinión publica de Latinobarometro que para el año 2017 involucra 20.000 entrevistas en 18 países latinoamericanos que representan a más de 600 millones de personas, donde se observa el desarrollo de las democracias utilizando indicadores de actitud, comportamiento y opinión. La encuesta Latinobarometro (2017) contribuye al estudio de ciertos indicadores relacionados a satisfacción de la democracia que podrían incidir en la variable dependiente confianza electoral a partir del modelo estadístico Ordeded probit, el cual permite comprender el grado proximidad de las variables. A partir de lo anterior, y de la revisión de fuentes teóricas se sostiene como hipótesis: a mayor satisfacción con la democracia, mayor confianza hacia los partidos políticos. $\mathrm{O}$ a la inversa entre menor satisfacción con la democracia, menor confianza hacia los partidos políticos, es decir que los resultados podrían presentarse de manera directa y proporcional.

Palabras ClaVe: partidos políticos, satisfacción con la democracia, vitalidad de los partidos, vigencia de los partidos.

\section{ABstract:}

The article is developed from the research question: What is the incidence of satisfaction with democracy in the trust towards political parties in Latin America, 2017? Which is approached based on data from the annual public opinion survey of Latinobarometro that by 2017 involves 20,000 interviews in 18 Latin American countries representing more than 600 million people, where the development of democracies is observed using indicators of attitude, behavior and opinion. The Latinobarometro survey (2017) contributes to the study of certain indicators related to the satisfaction of democracy that could influence the electoral confidence dependent variable based on the Ordeded probit statistical model, which allows us to understand the degree of proximity of the variables. The revision of theoretical sources is based on the hypothesis: the greater satisfaction with democracy, the greater confidence in political parties. Or conversely, the less satisfaction with democracy, the less confidence in political parties, that is, the results could be presented directly and proportionally.

KEYWORDS: political parties, satisfaction with democracy, vitality of parties, palidity of parties.

\section{INTRODUCCIÓN}

El presente artículo parte de una investigación de carácter cuantitativo, desarrolla un análisis estadístico del modelo Ordeded Probit que busca comprender la incidencia de indicadores de satisfacción de la democracia en la variable dependiente confianza electoral caracterizada en la encuesta anual de Latinobarometro realizada en el año de 2017. El propósito es comprender si existe una relación directamente proporcional entre las variables y con ello comprobar la teoría que sostiene el presente estudio.

En la actualidad existe una gran discusión teórica acerca de la necesidad o no de los partidos políticos en las sociedades democráticas y como estos contribuyen a la legitimidad de los procesos de participación ciudadana. Los debates giran en torno a la relación entre regímenes democráticos y partidos políticos como canales de representación. 
Pese a las dudas que se establecen en cuanto a la relación entre partidos políticos y regímenes democráticos en la modernidad, se puede decir que su relación se mantiene intacta, no se puede hablar de democracias sin partidos políticos. Los partidos políticos según Rafael Piñeiro y Fernando Rosenblatt (2017) se mantienen vitales en el accionar que ejercen dentro de los procesos interelectorales.

Siguiendo la línea de vitalidad de los partidos en las sociedades democráticas y profundizando más en su vigencia, autores como Rosenblatt (2018); Richard Gunther y José Ramón Montero (2003) resaltan la necesidad de los partidos políticos para el desarrollo de las democracias desde los procesos de adaptabilidad que puedan presentar en las sociedades cambiantes y modernizadas.

Los partidos políticos por tanto mantienen una relación contundente en el sostenimiento de las democracias actuales que es necesario estudiar a partir de su vitalidad y vigencia, lo cual permite comprender su grado de institucionalidad y la consolidación que tienen en las sociedades.

\section{A. Vitalidad de los partidos}

Los partidos políticos son un eje fundamental en los procesos de representación en las democracias actuales, dado que se mantienen activos en los distintos espacios interelectorales. Su papel como menciona Piñeiro y Rosenblatt (2017) no puede reducirse a las campañas políticas, los partidos políticos deben atraer a los militantes y mantener vivo su activismo a partir de la toma de decisiones en los gobiernos y en el diseño de políticas públicas, lo cual les permite preservar su expresión y reproducción en el campo político.

De esta manera, la vitalidad de los partidos políticos se relaciona a los procesos de satisfacción con la democracia al articularse a la complacencia de los ciudadanos por los canales de representación y participación política, lo cual incide en su confianza. La confianza hacia los partidos políticos les permite ganar legitimidad en las decisiones y negociaciones que se establecen en los gobiernos.

El respaldo político que puedan dar los ciudadanos a los partidos políticos, es fundamental en tanto mantienen viva su organización y aportan a la confianza hacia las instituciones públicas. La reproducción por tanto de las democracias y la confianza hacia los partidos políticos tiene que ver con la lealtad y compromiso de sus miembros.

Para la reproducción de las organizaciones partidarias es necesario que existan grupos de activistas que piensen tanto en sus intereses individuales (en su carrera), para los que el partido es un vehículo de la satisfacción de estos intereses, como en los objetivos colectivos de la organización (Piñeiro y Rosenblatt, 2017, p. 280).

Es importante por tanto contar con el apoyo de militantes leales en la toma de decisiones políticas, de esta forma los procesos de representación tendrán legitimidad y estabilidad institucional, lo cual aporta a la satisfacción por el régimen democrático y a su vez a la confianza hacia los partidos que activan la participación de los ciudadanos.

Siguiendo la línea de vitalidad de los partidos, es importante comprender su grado de institucionalización, dado que autores como Carlos Meléndez (2007) señalan que los partidos políticos que surgieron antes de la tercera ola de la democracia tenían mayor institucionalización e ideología. No obstante, se puede decir que en la actualidad los partidos aun persiguen intereses y tienen una identidad que puede influir en las decisiones de los votantes, lo cual es importante rescatar en los procesos de legitimidad que adelantan.

En las sociedades democráticas se puede establecer que existe una institucionalización en desarrollo de los partidos políticos, en tanto nuevos actores buscan ingresar al juego político a través del sistema electoral que les permite organizarse. El ingreso de nuevos actores al campo político significa que hay una conformidad y un reconocimiento de las reglas electorales que se establecen.

Si bien países de América Latina adelantan procesos de la institucionalización, es necesario reconocer que el desarrollo de partidos políticos presenta varios retos como son los cambios económicos y sociales, que 
influyen en la confianza de las personas por las instituciones, donde se mide la capacidad de resistencia y la recuperación de organizaciones a través del tiempo.

Asimismo, en los procesos de institucionalización de los partidos políticos, la confianza de las personas es el mayor reto que afrontan, dado que problemas de corrupción en las regiones de América Latina, influyen de manera negativa en las decisiones que se toman en los gobiernos. Igualmente, los problemas de corrupción influyen en las garantías que existen para el desarrollo de elecciones limpias, que ponen en riesgo los derechos civiles, sociales y políticos de las personas.

En temas de garantías de los derechos de las personas, Meléndez sostiene que es necesario fortalecer los partidos políticos a partir de regulaciones, "es necesario establecer regulaciones nacionales para favorecer la formación de los miembros de las agrupaciones políticas y sociales en los temas sustantivos de la política como la lucha contra la pobreza, la descentralización, reformas políticas, etc" (2007, p. 335).

La relación entre las sociedades democráticas y partidos políticos por tanto es directa, si los procesos de representación y participación funcionan bien, las personas tendrán mayor confianza hacia los partidos políticos y en las reglas de juego electoral. Lo cual les permitirá desarrollarse a lo largo del tiempo y legitimarse en las sociedades a partir de activistas leales.

Requiere la socialización de los integrantes de cada organización en los lineamientos elementales de la misma, así como la existencia de sistemas de comunicación de doble vía entre las dirigencias nacionales y la militancia a nivel nacional que aseguren la circulación fluida y transparente de la información (Meléndez 2007, p. 328).

De no contar con procesos que garanticen la confiabilidad por las instituciones democráticas la confianza hacia los partidos políticos será baja, lo cual afecta de manera negativa su legitimidad. De aquí la importancia de rescatar la transparencia en los procesos representativos que aporte a su consolidación y desarrollo.

\section{B. Vigencia de los partidos}

Extendiendo los argumentos que respaldan los estudios acerca de la vitalidad de los partidos políticos y profundizando más en su vigencia, es importante resaltar la necesidad de los partidos para el funcionamiento de las sociedades democráticas, a partir de procesos de actualización y adaptación. Autores como Gunther y Montero (2003) rescatan postulados de John Aldrich (1995) respecto al cambio de óptica de los estudios acerca de la descomposición de los partidos y apuestan a su revitalización.

Aldrich (1995) sugiere que los estudios relativos a «las tres Des» (decaimiento, declive y descomposición de los partidos) deben reemplazarse por «las tres Erres» (reaparición, revitalización y resurgimiento de los partidos), a la luz de los profundos cambios en las funciones (Gunther y Montero 2003, p. 16).

Las tres "erres" (reaparición, revitalización y resurgimientos) se basan en principios de confianza hacia los partidos políticos en las sociedades democrática actuales, cuyas practicas se refieren a procesos de adaptación a los cambios sociales, económicos y políticos y en la forma como los partidos asumen la incertidumbre que surge en torno a los resultados electorales.

En lo que se refiere a la incertidumbre como un factor que influye en los procesos de institucionalización de los partidos políticos, es importante destacar los postulados de Noam Lupu y Rachel Beatty (2012) respecto a que: la incertidumbre se encuentra presente en todas las sociedades. No obstante, en sociedades en desarrollo existe una mayor incertidumbre sobre las instituciones y los resultados electorales, lo cual se puede reducir al fortalecer las reglas

Las instituciones que se encuentran en riesgo por altos niveles de incertidumbre, pueden reducirla al crear reglas claras en el sistema electoral, "Una fuente final de incertidumbre en las democracias en desarrollo es institucional. Cuando la correspondencia entre reglas formales, instituciones informales, expectativas, y el comportamiento es alta, los estudiosos describen un sistema político como institucionalizado" (Lupu y Beatty, 2012, p. 1347). 
Por otra parte autores como Rosenblatt (2018) resaltan la vigencia de los partidos políticos a partir de su vibrancia, es decir su activación en los procesos de negociación y la motivación que ejercen en sus militantes. Los partidos vibrantes tienen que ver con la interacción de cuatro aspectos que son: propósito, trauma, canales de ambición y salidas moderadas.

El trauma activa la lealtad retrospectiva, mientras que el Propósito activa la lealtad prospectiva entre otros miembros de la sociedad. Canales de ambición promueven la actualización y mantiene al día el venir políticos sobre los beneficios potenciales de la afiliación de partido. Las barreras de salida evitan que los políticos ambiciosos deserten en los malos tiempos (Rosenblatt 2018, p. 48).

Los partidos vibrantes generan un apego de sus miembros y activan la participación de las personas en procesos políticos y públicos, expresando los ideales de los partidos y su puesta en marcha en el desarrollo de políticas públicas. La vibración de los partidos por tanto no es un proceso constante, sino que corresponde a un ciclo de vida organizacional.

En general el estudio de los partidos políticos es un aspecto clave para entender el funcionamiento de la democracia. Comprender el grado de institucionalidad de los partidos aporta a comprender la satisfacción por la democracia y por su parte la satisfacción con la democracia permite aproximaciones al grado de confianza que pueden tener las personas hacia los partidos políticos.

A partir de la revisión de teorías especializadas sobre partidos políticos se puede establecer que son varios los factores relacionados a la democracia que inciden en su confianza, dado que no son aspectos excluyentes sino complementarios que se retroalimentan constantemente.

Según la teoría de partidos, la confianza hacia instituciones públicas como es el congreso incide en su confianza, puesto que según Meléndez (2007) se relaciona a la ideología que manejan. La ideología de los partidos políticos se representa en los debates políticos donde existen diferentes posturas que aportan a la formulación de proyectos y políticas públicas.

La confianza en instituciones se relaciona de esta forma a los fines declarados y a procesos históricos que le dan una identidad a los partidos políticos, es decir tiene unos propósitos claros que defienden y los caracteriza como menciona Rosenblatt (2018), los cuales se establecen y expresan en los espacios de interacción de los partidos políticos en cada país.

Otro de los aspectos que se relaciona a la confianza hacia los partidos políticos, según autores como Piñeiro y Rosenblatt (2017) son los procesos de negociación que establecen los partidos políticos en la discusión de políticas públicas. Los ciudadanos se orientan al apoyo de partidos que buscan consensos como aporte al bienestar colectivo.

Por último, según la teoría es importante comprender que tanto confían los ciudadanos en el voto secreto y la institución electoral. Dado que señala el grado de creencia en las reglas de juego político que mencionan autores como Gunther y Montero (2003), lo cual reduce la incertidumbre y es un aspecto clave a tener en cuenta en los estudios de legitimidad y estabilidad de los partidos políticos.

De lo expuesto se plantea como hipótesis: $\mathrm{H} 1$ = A mayor satisfacción con la democracia mayor confianza hacia los partidos políticos.

De esta manera, según la revisión de teorías se espera que la confianza hacia los partidos políticos se pueda explicar a través de las siguientes variables relacionadas a la satisfacción con la democracia: satisfacción con el régimen; satisfacción con su funcionamiento; satisfacción con las negociaciones políticas; confianza en el voto; confianza en el congreso y confianza en la institución electoral.

\section{Metodología}

El presente trabajo se establece como un estudio empírico-analítico con un diseño explicativo, a partir de una base de datos de la encuesta de percepción sobre democracia realizada por Latinobarometro 2017. La cual contiene 20.200 observaciones y 324 variables de 18 países que conforman América Latina. 
De lo anterior, es necesario resaltar que se involucró a Brasil ya que forma parte de la región y se ha incluido en varios estudios de América Latina, pese a su diferencia en idioma. Se descartó a Guyana y Surinam dado que presentan diferencias en su cultura, idioma y organización política.

\section{Resultados}

\section{A. Estadísticas descriptivas}

En el apartado se presenta datos descriptivos acerca de la confianza hacia los partidos políticos; la satisfacción de los ciudadanos con el funcionamiento de la democracia y la relación entre satisfacción con la democracia y partidos políticos, esto con el fin de realizar una aproximación al tema de estudio.

En el grafico 1 se representa la confianza hacia los partidos políticos, donde la mayor parte de los ciudadanos con un 55\% no confían en los partidos, seguidamente un 29\% y $12 \%$ de las personas encuestadas dicen tener poca y algo de confianza en las instituciones partidistas. En general los datos señalan la baja confianza hacia los partidos políticos dado que apenas el $2 \%$ considera que confía mucho en ellos.

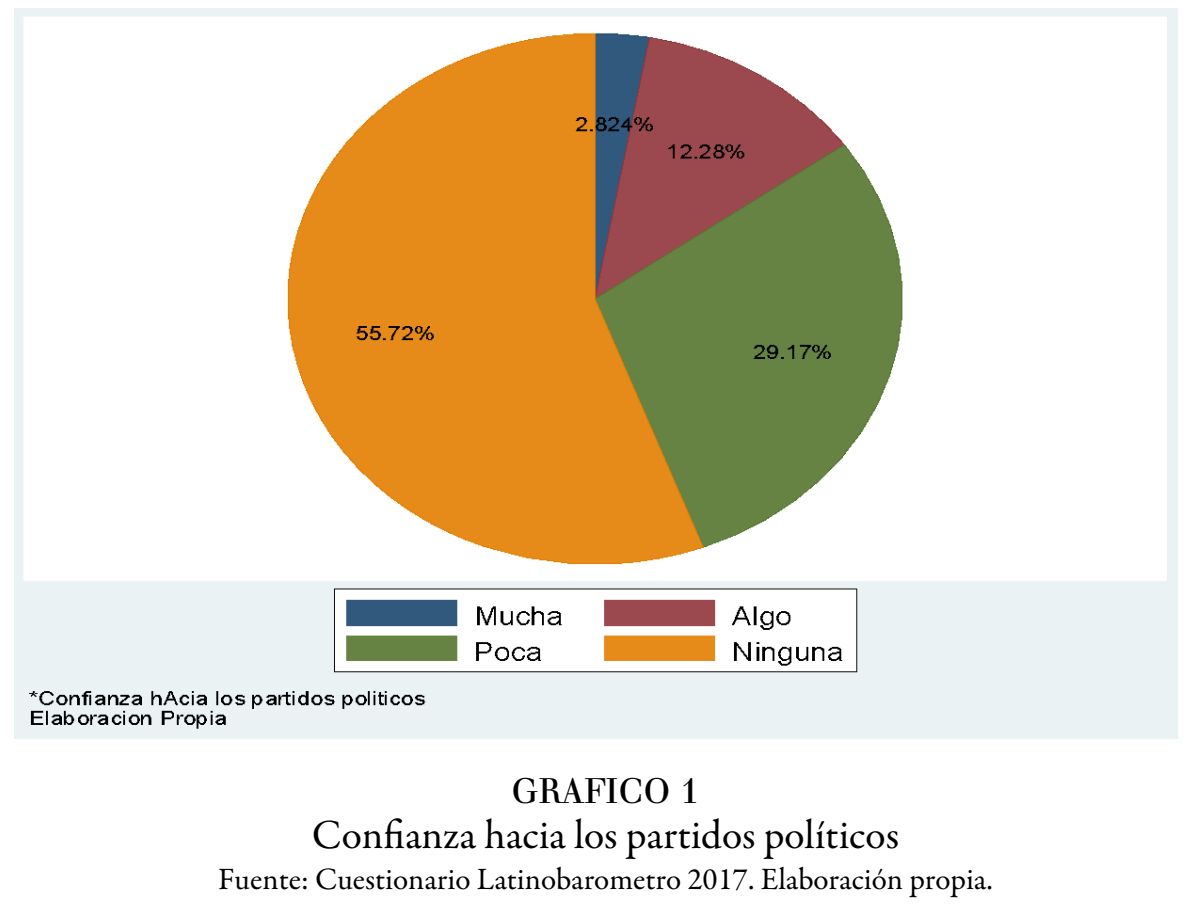

Respecto a la variable satisfacción con el funcionamiento de la democracia se observa en el grafico 2 que la mayor parte de las personas encuestadas con un $64 \%$ aproximadamente se encuentran algo satisfechos con el funcionamiento, lo cual señala que existen dudas de cómo se manejan los procesos políticos y públicos por parte de las instituciones del estado. En sí, no existe una conformidad por las instituciones que dirigen las democracias de los países de América Latina. 


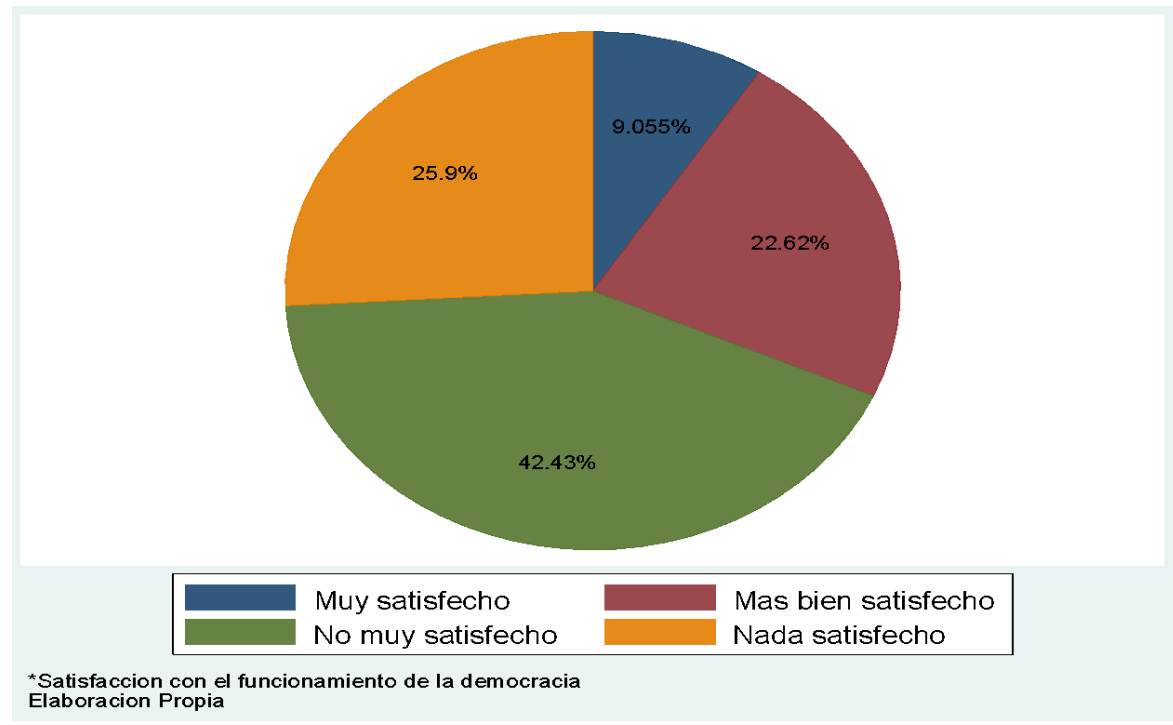

GRAFICO 2

Satisfacción con el funcionamiento de la democracia

Fuente: Cuestionario Latinobarometro 2017. Elaboración propia.

El gráfico 3 se presenta la relación entre la satisfacción con el funcionamiento de la democracia y la confianza hacia los partidos políticos, para lo cual se puede observar que en una escala del 0 al 5 las personas que tienen mayor satisfacción por el funcionamiento de la democracia, tienden a tener una mayor confianza hacia los partidos políticos y viceversa cuando existe menos satisfacción con la democracia, existe una mayor desconfianza por los partidos políticos.

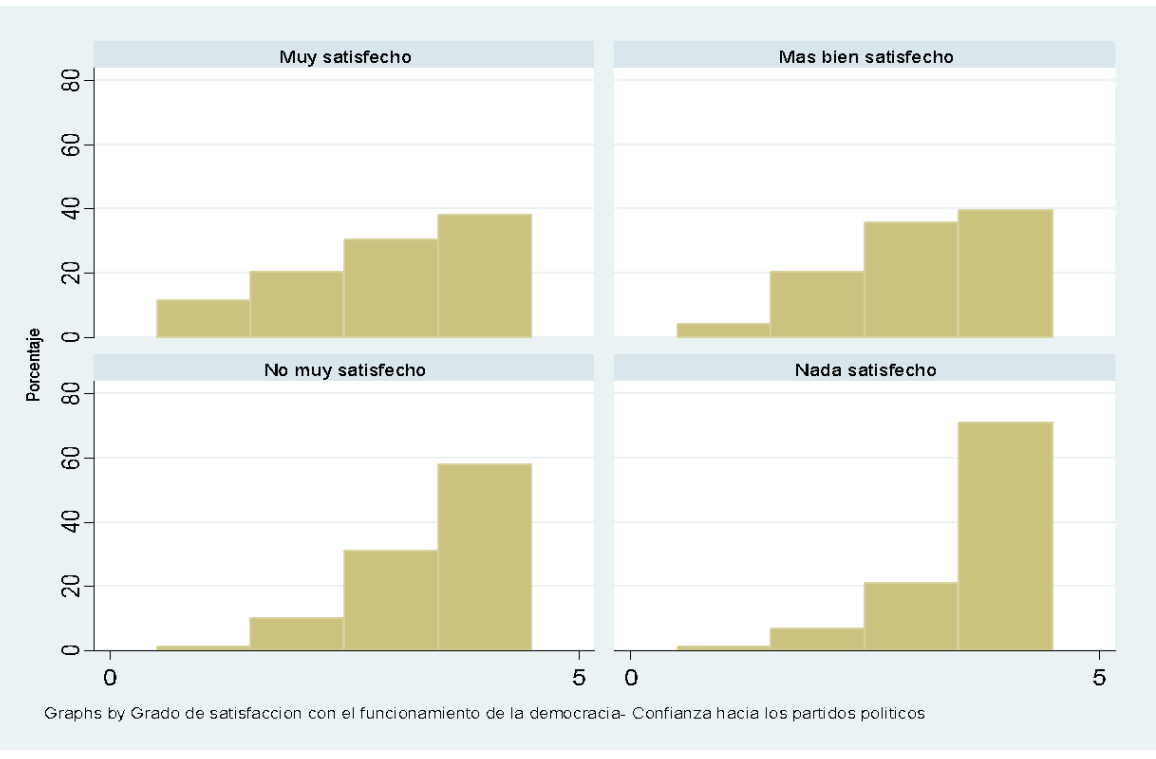

\section{GRÁFICO 3}

Satisfacción con la democracia- Confianza hacia los partidos políticos

Fuente: Cuestionario Latinobarometro 2017. Elaboración propia.

En términos generales se observa que existe una relación entre satisfacción con el funcionamiento de la democracia y confianza hacia los partidos políticos. Se observa que la mayor de las personas encuestadas presenta poca satisfacción por el funcionamiento de la democracia y así mismo una baja confianza hacia los partidos políticos. 


\section{B. Análisis estadístico}

Para la comprobación de hipótesis: a mayor satisfacción con la democracia, mayor confianza hacia los partidos políticos, se utilizó el modelo estadístico ordeded probit, puesto que la variable dependiente es ordinal (es decir que los valores presentan un orden natural como es mucho, algo, poco y ninguna). Para el análisis de datos, se utilizó el paquete estadístico STATA.

Los estimadores son los mismos para los parámetros del modelo, de modo que miden la confianza hacia los partidos políticos, a través de la siguiente ecuación:

$\mathrm{y}=\mathrm{Bo}+\mathrm{B} 1 \mathrm{x} 1+\mathrm{B} 2 \mathrm{x} 2+\mathrm{B} 3 \mathrm{x} 3+,,,, \mathrm{Bk} \mathrm{xk}+\mathrm{e}$

Dónde

y es la variable dependiente

Bo es el intercepto

B1 es el parámetro asociado con $x 1$, y así sucesivamente

$\mathbf{x} \mathbf{1}$ es variable independiente, ya si sucesivamente

e es el término de error

Ecuación del Modelo: y (confianza hacia los partidos políticos) = Bo + Satisfacción con el Régimen B1+ Funcionamiento Democracia B2 + Satisfacción con las negociaciones B3 + Confianza en el voto B4 + Confianza en el congreso B5 + Confianza institución electoral B6 + educación B7 + $\mathrm{e}^{*}$

Las variables proxy de satisfacción con la democracias que permiten explicar la confianza hacia los partidos políticos son: satisfacción con el régimen P8STGBS; satisfacción con su funcionamiento P9STGBSC_A; satisfacción con las negociaciones en el campo político P23STC; confianza en el voto P37NC; confianza en el congreso P14ST_D y confianza en la institución electoral P14ST_H. Se tuvo en cuenta como variable de control, educación S14 y se agregó la variable idenpa que permite observar la información por cada país.

El tratamiento de las variables fue convertir en datos faltantes, aquella información que contenía respuestas de no sabe/no responde. En relación a las variables P37NC confianza en el voto; P14ST.H confianza en la institución electoral y P14ST.D confianza en el congreso se transformaron a dicotómicas con valores de 1 y 0 , para lo cual se agruparon opciones de respuesta.

Finalmente se incluyó en el modelo la información por países, en primera instancia se convirtieron dummys por país, teniendo como base el país Argentina y luego se utilizó el comando de panel data xtset para formar panel por países. Al comparar los datos se observa que son semejantes por lo que se tiene en cuenta la variable i.idenpa, generada por el comando panel data. 
TABLA 1

Modelo ordeded probit

\begin{tabular}{|c|c|c|c|c|c|c|}
\hline P14ST_G & Coef. & Std. Err. & $\mathrm{Z}$ & $\mathrm{P}>\mathrm{z}$ & [ $95 \%$ Conf. & Interval] \\
\hline P8STGBS & .0443274 & .0116446 & 3.81 & 0.000 & .0215043 & .0671504 \\
\hline P9STGBSC_A & .2084782 & .0114337 & 18.23 & 0.000 & .1860686 & .2308878 \\
\hline P23STC & .0233001 & .0150596 & 1.55 & 0.122 & -.0062162 & .0528164 \\
\hline $\mathrm{p} 37 \mathrm{NC}$ & -.1524024 & .0210727 & -7.23 & 0.000 & -.1937041 & -.1111008 \\
\hline p14ST_H & -.7707923 & .0219328 & -35.14 & 0.000 & -.8137799 & -.7278048 \\
\hline p14ST_D & -.6703174 & .0229407 & -29.22 & 0.000 & -.7152802 & -.6253545 \\
\hline S14 & .0007297 & .0024261 & 0.30 & 0.764 & -.0040253 & .0054847 \\
\hline
\end{tabular}




\begin{tabular}{|c|c|c|c|c|c|c|}
\hline \multicolumn{7}{|l|}{ Idenpa } \\
\hline Bolivia & .1387777 & .0522648 & 2.66 & 0.008 & .0363406 & .2412149 \\
\hline Brasil & .3867789 & .0576091 & 6.71 & 0.000 & .2738671 & .4996907 \\
\hline Chile & .1767329 & .0541464 & 3.26 & 0.001 & .0706079 & .2828579 \\
\hline Colombia & .0963183 & .0527683 & 1.83 & 0.068 & -.0071057 & .1997422 \\
\hline Costa Rica & .4206581 & .0565385 & 7.44 & 0.000 & .3098447 & .5314715 \\
\hline $\begin{array}{l}\text { Rep. } \\
\text { Dominicana }\end{array}$ & .2789111 & .0569824 & 4.89 & 0.000 & .1672277 & .3905944 \\
\hline Ecuador & -.144611 & .0501582 & -2.88 & 0.004 & -.2429192 & -.0463028 \\
\hline E1 Salvador & .1218903 & .0579019 & 2.11 & 0.035 & .0084046 & .235376 \\
\hline Guatemala & .2280413 & .0595111 & 3.83 & 0.000 & .1114016 & .3446809 \\
\hline Honduras & -.0509768 & .0577571 & -0.88 & 0.377 & -.1641786 & .0622249 \\
\hline México & .2665032 & .0551711 & 4.83 & 0.000 & .1583699 & .3746366 \\
\hline Nicaragua & -.1273024 & .0583297 & -2.18 & 0.029 & -.2416265 & -.0129784 \\
\hline Panamá & .3853393 & .0593262 & 6.50 & 0.000 & .2690621 & .5016164 \\
\hline Paraguay & -.0650197 & .0531701 & -1.22 & 0.221 & -.1692312 & .0391919 \\
\hline Perú & .1092897 & .0533313 & 2.05 & 0.040 & .0047623 & .2138172 \\
\hline Uruguay & .1227253 & .0517407 & 2.37 & 0.018 & .0213154 & .2241353 \\
\hline Venezuela & -.4056694 & .0514198 & -7.89 & 0.000 & -.5064504 & -.3048883 \\
\hline
\end{tabular}

Fuente: Cuestionario Latinobarometro 2017. Elaboración propia. 
El modelo estadístico señala que los regresores incluidos son significativos a excepción de negociaciones de los partidos en el campo político P23STC y educación S14, cuyo p $>\mathrm{z}$ es mayor a 0.05. Los datos en general permiten comprobar la hipótesis de estudio dado que la satisfacción con el régimen democrático y con su funcionamiento incide directamente en la confianza hacia los partidos políticos, es decir que cuando existe mayor satisfacción por la democracia existe mayor confianza hacia los partidos políticos y viceversa.

En el caso de América Latina pese a que se observa una relación directa entre satisfacción con la democracia y confianza hacia los partidos políticos, es importante resaltar que la satisfacción por el régimen y la confianza hacia los partidos políticos es baja según se pudo ver en las estadísticas descriptivas. La incertidumbre que rodea el funcionamiento de las democracias afecta la estabilidad y legitimidad que pueden dar los militantes a sus partidos como señalan Piñeiro y Rosenblatt (2017).

En lo que se refiere a la satisfacción con la democracia y su incidencia en la confianza hacia los partidos políticos por países, se puede observar que las personas pertenecen a Ecuador, Honduras, Nicaragua, Paraguay y Venezuela, presentan menos probabilidad de confiar en los partidos políticos, al mantener coeficientes negativos en relación a la variable dependiente. No obstante, Honduras y Paraguay mantienen $\mathrm{p}>\mathrm{z}$ superior a 0.05 , por lo tanto no son significativos para explicar la variable dependiente.

En relación a los regresores de satisfacción con la democracia se puede ver que variables como: p37NC $=$ confianza en él voto, p14ST_H $=$ confianza en la institución electoral y p14ST_D $=$ confianza en el congreso, se relacionan de manera negativa con la variable confianza hacia los partidos políticos.

Variables como confianza por el voto secreto y confianza por la institución electoral señalan la baja confianza por las instituciones de la democracia, esto se relaciona a la falta de reglas claras que según autores como Gunther y Montero (2003), causa problemas de estabilidad a los partidos dado que en muchas ocasiones se han visto implicados en problemas de corrupción que afectan su imagen y desempeño.

Por último, la baja confianza de las personas hacia el congreso, es decir hacia el espacio de interacción de los partidos políticos puede estar relacionado a la baja ideología que perciben las personas de los partidos actuales y la falta de convicción por los fines declarados que tratan autores Meléndez (2007) y Rosenblatt (2018).

\section{Conclusiones}

El estudio establece que la satisfacción con el funcionamiento de la democracia se relaciona de forma directa con la confianza hacia los partidos políticos. Para el caso de América Latina, si bien existe una satisfacción por la democracia y una confianza hacia los partidos políticos, se puede decir que es baja.

Según los resultados del estudio existe una baja confianza hacia las instituciones de las democracias, lo cual afecta la legitimidad de los partidos políticos, esto demuestra que problemas de corrupción reducen la confianza de las personas por los procesos electorales y aumenta su incertidumbre hacia los resultados, siendo un factor negativo para la reproducción de las democracias.

Las instituciones de la democracia como es la electoral y el congreso se relacionan de forma negativa con la confianza hacia los partidos políticos. De esta forma, son factores a tener en cuenta para el fortalecimiento de las democracias, a partir de reglas claras que incentiven la confianza hacia los procesos democráticos.

Finalmente es importante que se desarrollen estudios que profundicen en torno a la confianza de las personas por las instituciones de la democracia como son los partidos políticos, esto con el fin de generar diagnósticos de las democracias actuales como aporte a los procesos de fortalecimiento de las instituciones.

\section{Referencias}

Aldrich, J. H. (1995). Why parties?: The origin and transformation of political parties in America. University of Chicago Press. 
Gunther, R. y Montero, J. (2003). “Los estudios sobre los partidos políticos: una revisión crítica”. Working Paper 12/2003. Madrid: Colección en la Red de Cuadernos de Trabajo.

Lupu, N. \& Beatty R. (2012). "Political parties and uncertainty in developing democracies." Comparative Political Studies 46(11), pp. 1339-1365.

Meléndez, C. (2007). “Análisis comparado de las agrupaciones políticas de los Países Andinos”. En Roncagliolo, R. \& Meléndez, C. (comp.). La politica por dentro. Lima: Ágora Democrática-IDEA-Transparencia. (pp. 41-74).

Piñeiro, R., \& Rosenblatt, F. (2017). “Tipos de activistas en organizaciones partidarias”. Politica y gobierno, 24(2), pp. 275-300.

Rosenblatt, F. (2018). Party Vibrancy and Democracy in Latin America. New York: Oxford University Press. (pp. 3-73).

CC BY-NC-SA 